\title{
Wilms' tumor 1 antigen immunoreactivity in epithelial ovarian cancer - diagnostic and prognostic value
}

\author{
Elzbieta Zarychta ${ }^{1}$, Katarzyna Lepinay ${ }^{1}$, Sebastian Szubert ${ }^{1}$, Jakub Jozwicki ${ }^{2}$, \\ Jan Misiak ${ }^{3}$, Anna A. Brozyna ${ }^{4}$, Katarzyna Kosinska-Kaczynska ${ }^{1}$, Agnieszka Lewandowska ${ }^{1}$, \\ Ewa Malicka ${ }^{1}$, Adrianna Makarewicz ${ }^{5}$, Piotr Rhone ${ }^{6}$, Wojciech Jozwicki ${ }^{3}$
}

${ }^{1}$ Second Department of Obstetrics and Gynecology, Centre of Postgraduate Medical Education, Warsaw, Poland

${ }^{2}$ Department of Clinical Pathomorphology, Faculty of Medicine, Ludwik Rydygier Collegium

Medicum in Bydgoszcz, Nicolaus Copernicus University, Torun, Poland

${ }^{3}$ Department of Tumor Pathology and Pathomorphology, Department of Oncology, Faculty of Health Sciences, Ludwik Rydygier Collegium Medicum in Bydgoszcz, Nicolaus Copernicus University, Torun, Poland

${ }^{4}$ Department of Human Biology, Institute of Biology, Faculty of Biological and Veterinary Sciences, Nicolaus Copernicus University, Torun, Poland

${ }^{5}$ Department of Oncology, The Franciszek Lukaszczyk Oncological Center, Bydgoszcz, Poland ${ }^{6}$ Department of Clinical Breast Cancer and Reconstructive Surgery, The Franciszek Lukaszczyk Oncological Center, Bydgoszcz, Poland

\begin{abstract}
Objectives. Ovarian cancer is a heterogeneous disease, with a number of different histological subtypes with various responses to treatment. Wilms' tumor 1 (WT1) immunoreactivity is used to distinguish between OC's various subtypes. However, little is known about the protein's role as a prognostic factor. Thus, the main aim of our study was to evaluate the relationship between WT1 expression and patient overall survival (OS) and lymph node metastases.

Materials and methods. Study group consisted of 164 women aged 22-84, diagnosed with epithelial ovarian cancer (EOC). WT1 expression in histological slides was assessed by immunohistochemistry.

Results. Serous tumors were the most common subtype among EOC $(\mathrm{n}=126 ; 76.8 \%)$, followed by endometrioid $(\mathrm{n}=20 ; 12.2 \%)$, clear-cell $(\mathrm{n}=14 ; 8.5 \%)$ and mucinous cancer $(\mathrm{n}=4 ; 2.4 \%)$. Of all serous EOC, WT1-positive tumors accounted for $75.6 \%$ of cases and this number was significantly higher than in other histological subtypes $(\mathrm{p}<0.0001)$. Patients with lymph node metastases were more likely to have WT1-positive than WT1-negative tumors $(\mathrm{p}=0.006)$. There was no significant correlation between WT1 immunoreactivity and OS across the whole study group of EOC patients $(p=0.6)$; however, in the group of non-serous (mucinous, endometrioid and clear-cell) EOC subjects, WT1 immunoreactivity was associated with shorter OS ( $p=0.046)$.

Conclusions. WT1 immunoreactivity may be helpful in differentiating primary epithelial serous carcinomas from non-serous ovarian cancers; however, its prognostic role in EOC is rather uncertain. (Folia Histochemica et Cytobiologica 2020, Vol. 58, No. 3, 198-207)
\end{abstract}

Key words: epithelial ovarian cancer; WT1; Wilms' tumor antigen 1; lymph node metastases

\footnotetext{
Correspondence address: Prof. Wojciech Jozwicki, MD., PhD

Department of Tumor Pathology and Pathomorphology,

Department of Oncology, Faculty of Health Sciences,

Ludwik Rydygier Collegium Medicum in Bydgoszcz, Nicolaus

Copernicus University in Torun, Torun, Poland

2 Romanowska St., Bydgoszcz 85-796, Poland

e-mail: jozwickiw@co.bydgoszcz.pl
} 


\section{Introduction}

In 2018 over 295000 women worldwide were diagnosed with ovarian cancer (OC) and almost 185000 women died because of this malignancy, making it the eight most common cause of cancer death in females [1]. Well-established risk factors of ovarian cancer are a family history of ovarian or breast cancer, BRCA1 and BRCA 2 mutations, Lynch syndrome, hereditary non-polyposis colon cancer (HNPCC), nulliparity or low parity, use of hormone replacement therapy, menarche at an early age, greater height, being overweight, tobacco smoking and diagnosis of endometriosis [2,3]. Ovarian cancer is a highly heterogeneous disease that involves malignancies with various histological features, site of origin, grade, risk factors, prognoses and treatment. According to tumor cell histology, the main types of epithelial ovarian cancer are serous $(52 \%)$, endometrioid $(10 \%)$, mucinous $(6 \%)$ and clear-cell $(6 \%)$. About one quarter of epithelial ovarian cancers are classified as either unspecified or rarer subtypes of ovarian cancer. Non-epithelial cancers make up approximately $10 \%$ of all ovarian neoplasms and are of secondary importance [4].

Wilms' tumor 1 (WT1) is a transcription factor involved in the regulation of genes such as growth factors, regulators of cell cycle and apoptosis. Primarily regarded as a tumor suppressor gene, it is now considered to have oncogenic functions. WT1 is located on chromosome 11 and its expression is found in normal human tissues such as the kidney, ovary, testis, spleen, peritoneal mesothelium and fallopian tube epithelium, as well as in leukemias (where WT1 mRNA expression levels increase along with progression of the disease) and various types of solid tumors, including ovarian cancer. WT1 gene expression levels in many tumors may serve as a prognostic factor. Previous studies demonstrated that in leukemias, as well as in solid cancer cells, induction of WT1 expression promoted cell growth and motility together with suppression of apoptosis [5-10].

Most patients with epithelial ovarian cancer (EOC) present with advanced stage disease. Prognosis is strongly associated with the disease stage at the time of diagnosis, and the prognosis also differs largely according to epithelial subtype. Serous carcinomas are diagnosed mostly at stage III $(51 \%)$ or IV $(29 \%)$, while more than half $(58-64 \%)$ of endometroid, mucinous, and clear cell carcinomas are diagnosed at stage I. Apart from stage, other factors associated with poor prognosis are high histological grade, patient age of 65 years or older, large volume of the residual tumor, and low global quality-of-life score. However, while WT1's role in distinguishing serous
EOC from other subtypes of ovarian cancer seems to be established, there are still discrepancies regarding its role in prognosis in both serous and non-serous EOC [4, 11-14].

The aims of this study were to establish whether the presence of WT1 immunoreactivity in ovarian cancer cells varies between particular histological subtypes of the malignancy, to determine whether it is associated with lymph node metastases, and to assess its prognostic value.

\section{Material and methods}

Patient samples and clinical data. Our study group comprised 164 women aged 22 to 84 (age $59.45 \pm 11.27$; mean $\pm \mathrm{SD})$ diagnosed with EOC. All patients were operated on between March 2010 and March 2018 in the Clinical Department of Gynecological Oncology, The Franciszek Lukaszczyk Oncological Center Bydgoszcz, Poland; and each patient underwent a comprehensive histopathological confirmation of the diagnosis along with an analysis of WT1 immunoreactivity in cancer cells. Disease stage was assigned in accordance with the International Federation of Gynecology and Obstetrics (FIGO) surgical staging criteria. The histologic grade was categorized as either high or low. The clinicopathological characteristics of the study group are shown in Table 1.

Patients underwent longitudinal laparotomy extending from the xiphoid process to the pubic bone. All of the patients underwent bilateral/unilateral salpingoophorectomy and pelvic peritonectomy with retroperitoneal hysterectomy or, in the case of previous hysterectomy, vaginal vault resection. Additionally, total omentectomy was performed. Appendectomy was performed in cases of tumor infiltration or where there was suspicion of the mucinous type of ovarian cancer. Lymphadenectomy was always performed in cases where enlarged or suspicious lymph nodes were found. In cases where the lymph nodes were unchanged, the primary surgeon decided whether to perform lymphadenectomy. The resection of other organs was performed when necessary, depending on the degree of tumor infiltration, in order to remove all macroscopic lesions. All surgeries were performed by accredited gynecological oncologists (in most cases, L.W.). The extent of post-surgery residual disease was described according to Sugarbaker score [15]. All patients had received first-line chemotherapy consisting of intravenous carboplatin and paclitaxel.

The present study was approved by the Human Research Ethics Committee of Centre of Postgraduate Medical Education in Warsaw $(8 / \mathrm{PB} / 2020)$. The study was conducted in accordance with the ethical standards and principles embodied in the Declaration of Helsinki. All subjects provided written informed consent to participate in this study after receiving a full explanation of the aim of the study. 
Table 1. Clinicopathological characteristics of the study group

\begin{tabular}{|c|c|c|c|c|c|}
\hline Type of EOC & $\begin{array}{l}\text { Serous } \\
\mathbf{N}\end{array}$ & $\begin{array}{l}\text { Mucinous } \\
\mathbf{N}\end{array}$ & $\begin{array}{c}\text { Endometrioid } \\
\mathbf{N}\end{array}$ & $\begin{array}{c}\text { Clear cell } \\
\quad \mathbf{N}\end{array}$ & $\begin{array}{c}\text { Total } \\
\text { N }\end{array}$ \\
\hline Number of patients & 126 & 4 & 14 & 20 & 164 \\
\hline Mean age & 59.56 & 54 & 60.15 & 59.42 & 59.45 \\
\hline \multicolumn{6}{|l|}{ Lymph nodes: } \\
\hline Positive & 29 & 0 & 1 & 2 & 32 \\
\hline Negative & 97 & 4 & 13 & 18 & 132 \\
\hline \multicolumn{6}{|l|}{ Grade } \\
\hline Low grade & 1 & 1 & 0 & 3 & 2 \\
\hline High grade $(\mathrm{G} 2, \mathrm{G} 3)$ & 125 & 3 & 14 & 17 & 162 \\
\hline \multicolumn{6}{|l|}{ FIGO stage } \\
\hline I & 14 & 0 & 1 & 4 & 19 \\
\hline II & 11 & 0 & 4 & 6 & 21 \\
\hline III & 91 & 3 & 9 & 10 & 113 \\
\hline IV & 10 & 1 & 0 & 0 & 11 \\
\hline \multicolumn{6}{|l|}{$\mathrm{CC}$} \\
\hline 0 & 75 & 2 & 8 & 16 & 101 \\
\hline 1 & 15 & 0 & 2 & 0 & 17 \\
\hline 2 & 22 & 1 & 3 & 3 & 29 \\
\hline 3 & 14 & 1 & 1 & 1 & 17 \\
\hline
\end{tabular}

Abbreviations: EOC - epithelial ovarian cancer; CC — completeness of cytoreduction according to Sugarbaker score [15].

Immunohistochemistry. Immunohistochemistry staining was performed with mouse monoclonal WT1 antibody (Roche/Ventana, Oro Valley, AZ, USA, 6F-H2 clone, \#760-4397) and an ultraView Universal DAB Detection Kit chromogen (Roche/Ventana, \#760-500) using a Benchmark Ultra instrument (Roche/Ventana).

Staining of all samples was done on $4 \mu \mathrm{m}$ tissue sections which were obtained from tumor samples fixed in $4 \%$ buffered formaldehyde and embedded in paraffin blocks according to a standard protocol. Paraffin block sections were placed on adhesive slides (Knittel Glass) and incubated for $2 \mathrm{~h}$ at $60^{\circ} \mathrm{C}$ in a thermostatic chamber.

All the steps of the IHC staining were automated using a Benchmark Ultra instrument (Roche/Ventana). The final step involved dehydration in a series of ethanol concentrations and xylene followed by a coverslip mounting medium (Consul Mount Shandon, Thermo Scientific, Kalamazoo, MI, USA). Each patient sample series included a control sample as recommended by the manufacturer. In each case, the level of WT1 expression was assessed as either negative or positive (see Figs. 1B, 1D, and 1F, $1 \mathrm{H}$ respectively). WT1 expression was assessed independently by two pathologists. The histological classification of the tumors was performed according to the WHO Classification of Tumors.

Microscopic assessment was performed with the use of a Nikon Eclipse 80i microscope (Nikon, Tokyo, Japan).
Pictures were taken with a Nikon Digital Sight DS Fi1-U2 camera and with NISElements BR 3.0 software (Nikon Instruments Europe B.V., Badhoevedorp, The Netherlands).

Statistical analysis. Categorical data were analyzed using the Fisher's exact test, and with the Freeman-Halton extension when appropriate. We performed multiple-regression model with stepwise entering method to evaluate the relationship between WT1 immunoreactivity and clinicopathological features of EOC disease. Survival analysis was conducted using Kaplan-Meier survival curves. The log-rank test was used for the comparing survival distributions between the analyzed subgroups. P-values $<0.05$ were considered significant.

\section{Results}

\section{WT1 immunoreactivity in EOC}

A total of 164 subjects were included in our study. The most common type of ovarian epithelial cancer in the study group was serous $(\mathrm{n}=126 ; 76.8 \%)$, followed by endometrioid $(n=20 ; 12.2 \%)$, clear cell $(n=14$; $8.5 \%)$ and mucinous $(\mathrm{n}=4 ; 2.4 \%)$. The number of WT1-positive and negative tumors varied significantly between the particular types of EOC and the data are shown in Table $2(\mathrm{p}<0.0001)$. When we compared serous and non-serous EOC, we have found 
HE

WT1

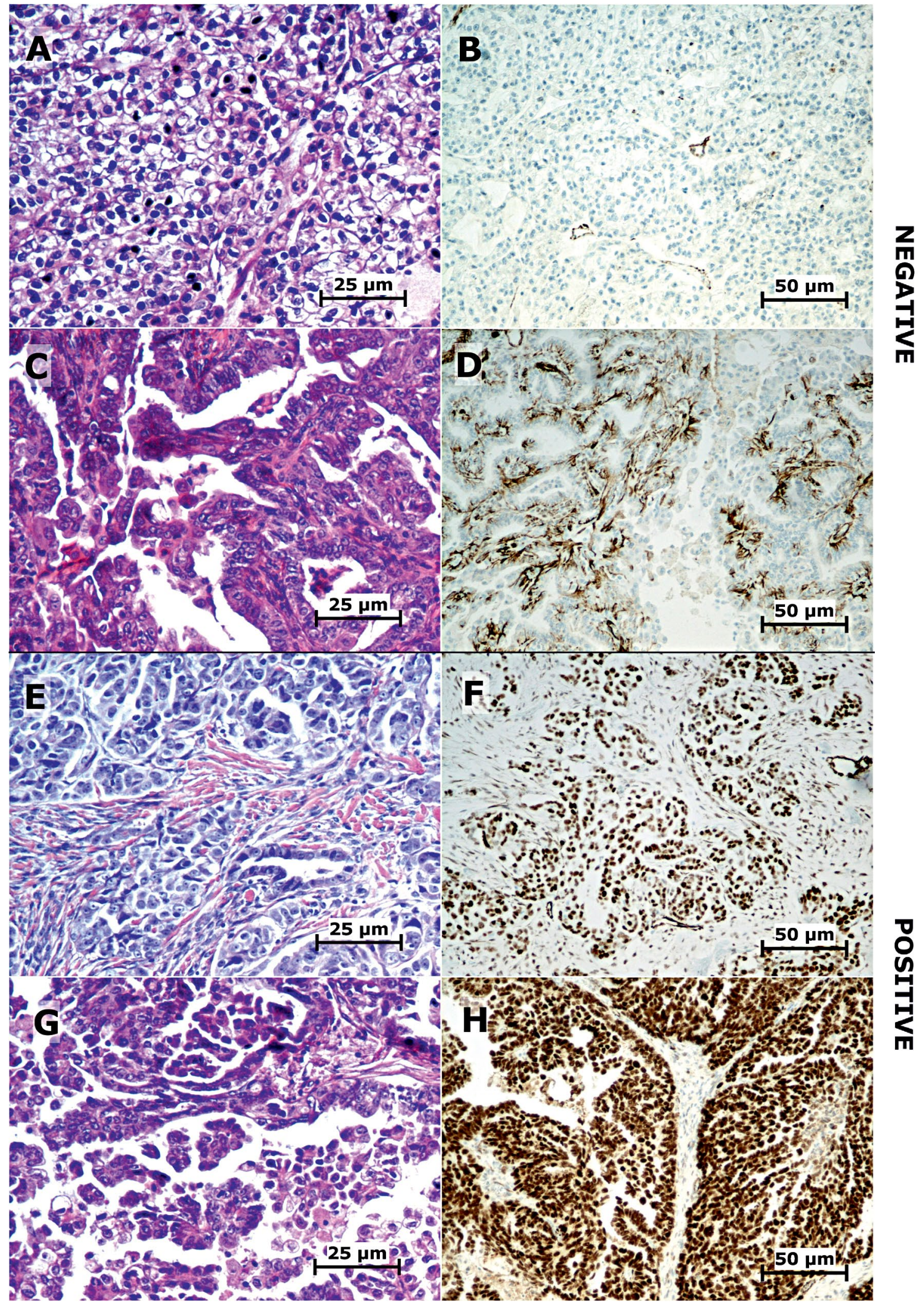

Figure 1. Representative staining intensity of WT1 in ovarian cancer (OC) cells, defined as either negative (no cellular expression (B) or with membrane reactivity but without nuclear expression (D)), or positive (low and mediate (F) or high (H) expression), regardless of the histological type of cancer (A - clear-cell type, C - endometrioid type, E and G - serous type). 
Table 2. WT1 immunoreactivity in different histological types of EOC

\begin{tabular}{|l|c|c|c|c|c|c|}
\hline Type of EOC & $\begin{array}{c}\text { Serous } \\
\mathbf{N}(\boldsymbol{\%})\end{array}$ & $\begin{array}{c}\text { Mucinous } \\
\mathbf{N}(\boldsymbol{\%})\end{array}$ & $\begin{array}{c}\text { Clear-cell } \\
\mathbf{N}(\boldsymbol{\%})\end{array}$ & $\begin{array}{c}\text { Endometrioid } \\
\mathbf{N}(\boldsymbol{\%})\end{array}$ & $\begin{array}{c}\text { Total } \\
\mathbf{N}(\boldsymbol{\%})\end{array}$ & p-value \\
\hline WT1 $(+)$ & 106 & 0 & 9 & 9 & 124 & \\
\hline WT1 $(-)$ & 20 & 4 & 5 & 11 & 40 & P $<0.0001$ \\
\hline Total & $126(76.83)$ & $4(2.44)$ & $14(8.54)$ & $20(12.19)$ & $164(100)$ & \\
\hline
\end{tabular}

Abbreviations: WT1 — Wilms' tumor; EOC — epithelial ovarian cancer.

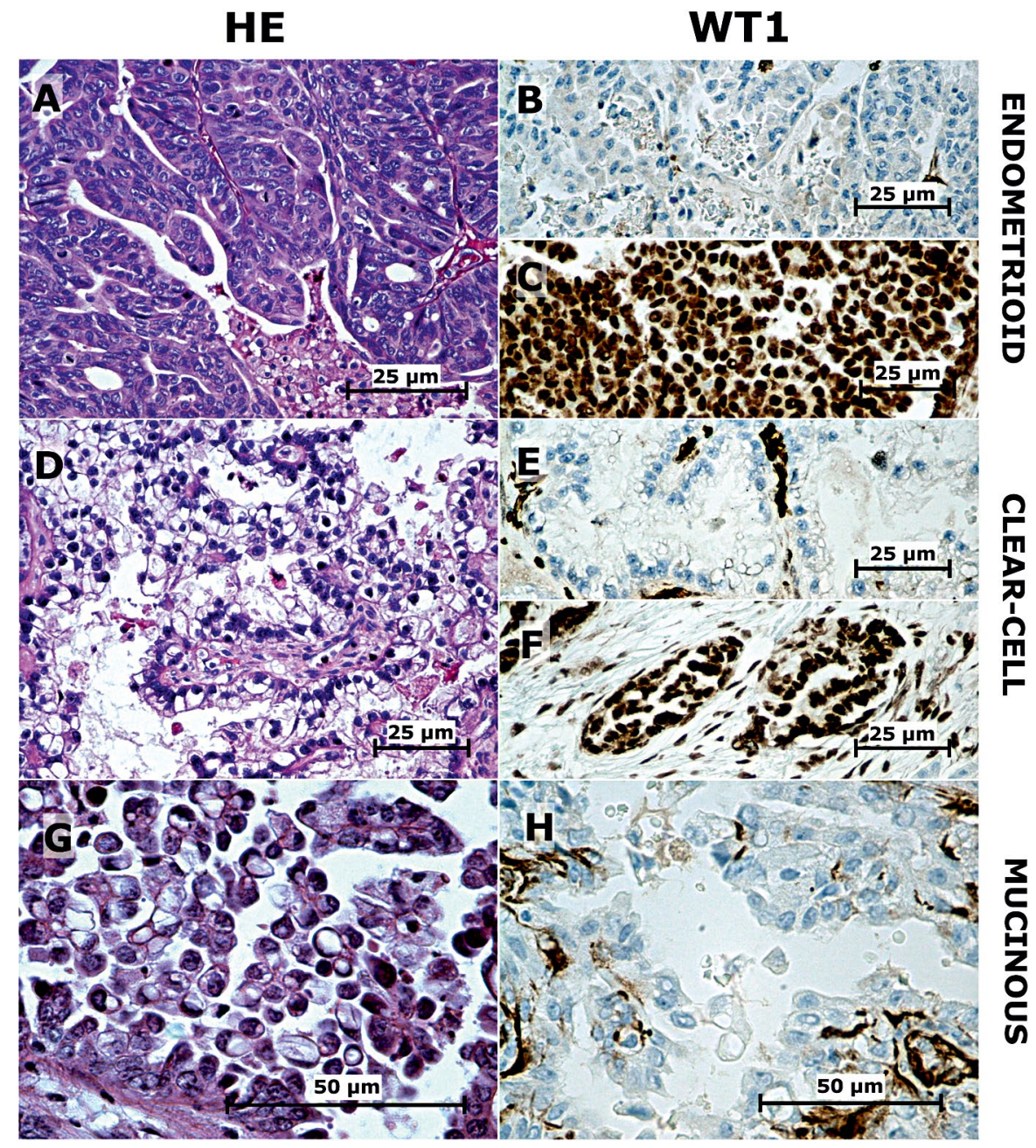

Figure 2. The complete absence of WT1 nuclear expression was observed in mucinous ovarian cancers (G-H). In contrast, endometrioid (A) and clear cell (D) carcinomas varied in terms of the intensity of WT1 expression (B vs. C and E vs. F).

significantly higher WT1 immunoreactivity in serous EOC $(84 \%$ vs. $47 \%, \mathrm{P}<0.0001)$. In the multiple regression model including the following independent variables: histopathological type of the tumor (serous $v s$. non-serous), post-surgery residual disease, lymph node metastases; only the tumor histopathological type $(\mathrm{P}<0.0001)$ was significantly associated with WT1 immunoreactivity.
The presence or absence of expression was observed in all non-serous ovarian cancers except mucinous (Fig. 2).

\section{WT1 immunoreactivity and lymph nodes metastases}

Furthermore, we analyzed the histological types of ovarian cancer and WT1 immunoreactivity in patients with lymph node metastases. In general, metastatic 
Table 3. Different histological types of EOC in WT1-positive and WT1-negative patients with lymph node metastases

\begin{tabular}{|l|c|c|c|c|}
\hline Type of EOC & $\begin{array}{c}\text { Serous } \\
\mathbf{N}\end{array}$ & $\begin{array}{c}\text { Non-serous } \\
\mathbf{N}\end{array}$ & $\begin{array}{c}\text { Total } \\
\mathbf{N}\end{array}$ & p-value \\
\cline { 1 - 3 } WT1-positive & 28 & 2 & 30 & \multirow{2}{*}{$\mathrm{P}=0.2$} \\
\hline WT1-negative & 1 & 1 & 2 & \\
\hline
\end{tabular}

Abbreviations as for table 2.

Table 4. Presence of lymph node metastases in WT1-positive patients $v s$. WT1-negative patients

\begin{tabular}{|l|c|c|c|c|}
\hline Presence of lymph nodes metastases & $\begin{array}{c}\text { LN (+) } \\
\text { N (\%) }\end{array}$ & $\begin{array}{c}\text { LN (-) } \\
\text { N (\%) }\end{array}$ & $\begin{array}{c}\text { Total } \\
\text { N (\%) }\end{array}$ & p-value \\
\cline { 1 - 3 } WT1-positive & $30(24.19)$ & $94(75.81)$ & $124(100)$ & \multirow{2}{*}{ P $=0.006$} \\
\hline WT1-negative & $2(5.0)$ & $38(95.00)$ & $40(100)$ & \\
\hline
\end{tabular}

Abbreviations: WT1 — Wilms' tumor; LN — lymph nodes.

lymph nodes were confirmed in $32(19.5 \%)$ patients. In the serous tumors, the metastatic spread of the disease was found in $23 \%$ of the cases; in the clear cell cancers in $14.3 \%$ of the cases; in the endometrioid in $5 \%$ of the cases; and in the mucinous ovarian cancers there were no cases of metastatic lymph nodes. In the group of both WT1-positive and WT1-negative patients with metastatic lymph nodes, the presence of lymph node metastases did not differ significantly according to the type of cancer, whether serous or non-serous, $\mathrm{p}=0.2$ ). The results are shown in Table 3 .

Subsequently, statistical calculations were made on the study group data based on the presence of lymph node metastases and WT1 immunoreactivity. Patients with WT1-positive tumors were more likely to have lymph node metastases; specifically, 30 out of $124(24.2 \%)$ WT1-positive patients had affected LN compared with 2 out of $40(5 \%)$ WT1-negative patients (Table 4). The differences between these groups were statistically significant $(p=0.006)$.

\section{Survival analysis}

The correlation between WT1 immunoreactivity and overall survival (OS) in patients with epithelial ovarian cancer was evaluated via Kaplan-Meier curves. Survival analysis included 164 EOC patients: 124 women with WT1 immunoreactivity and 40 without WT1 immunoreactivity. The median OS for the first group was 1084 days, while the median OS in second group was not reached. The presence of WT1 immunoreactivity in the tumor cells did not correlate with a shorter OS (HR $(95 \%$ CI) $=1.2594(0.7746-2.0477), \mathrm{p}=0.4)$ in members of the group comprising all ovarian cancer patients (Fig. 3A).

Finally, the prognostic value of WT1 in ovarian cancer with different histological subtypes was as- sessed. Patients were divided into two subgroups: serous and non-serous (mucinous, endometrioid and clear-cell). Among serous ovarian cancer patients with the presence of WT1 in cancer cells, OS was shorter than among WT1-negative patients; however, the difference was not statistically significant (HR $(95 \% \mathrm{CI})$ $=0.8643(0.4389-1.7020), P=0.6)]$ (Fig. 3B). On the other hand, in the non-serous group, WT1-positive patients presented with a significantly shorter OS than the WT1-negative patients $(\mathrm{HR}(95 \% \mathrm{CI})=2.6400$ (1.0042-6.9404), $\mathrm{P}=0.046$ ) (Fig. 3C).

\section{Discussion}

In our study, we investigated WT1 immunoreactivity in different histological subtypes of ovarian cancer as well as its association with the presence of lymph node metastases. We analyzed the overall survival rates of the whole study group of WT1-positive and WT1-negative patients as well as considering the OS rates for serous and non-serous tumors.

Various studies have found WT1-positivity in serous ovarian tumors to range between 61 and 97\% [16-21]. The percentage of ovarian serous carcinoma expressing WT1 in our study (84\%) fell within the range of values reported in those studies. Furthermore, in our study we found no WT1-positive mucinous tumors, which was a similar result to those of other authors $[18,19,21]$. However, there were discrepancies between our study and others' concerning endometrioid and clear-cell EOCs. We found WT1 immunoreactivity in $64 \%$ of clear-cell ovarian carcinomas, which diverges from the results obtained by Hylander et al. (20\% of WT1-positive clear-cell carcinomas) [16] and Acs et al. (22\% of WT1-positive 


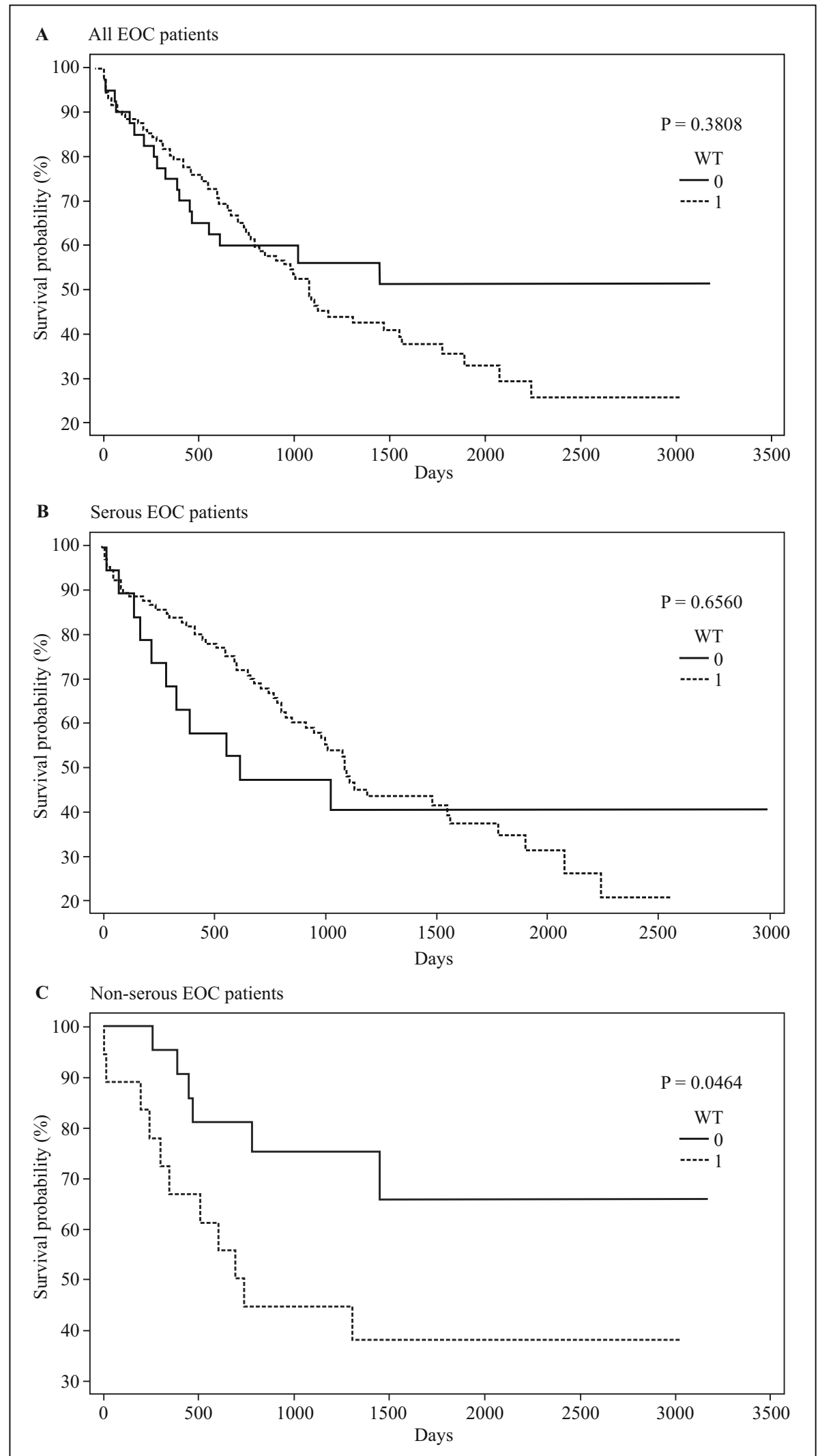

Figure 3. Kaplan-Meier survival analysis in epithelial ovarian cancer (EOC) in relation to Wilms' tumor (WT1) immunoreactivity. A. Overall survival of the all EOC patients: WT1 $(-)$ group $(\mathrm{n}=40$, median overall survival (mOS) was not reached) vs. WT1 $(+)$ group $(\mathrm{n}=124, \operatorname{mOS} 1084$ days $), \mathrm{P}=0.4$. B. Overall survival profile of the serous EOC patients: WT1 (-) group $(\mathrm{n}=19$, mOS 614 days) vs. WT1 ( + ) group $(\mathrm{n}=106$, mOS 1084 days), $\mathrm{P}=0.7$. C. Overall survival profile of the non-serous EOC patients: WT1 (-) group $(\mathrm{n}=21$, mOS was not reached) $v$. WT1 $(+)$ group $(\mathrm{n}=18$, mOS 697 days $), \mathrm{P}=0.046$. 
clear-cell carcinomas) [18]. Also, in studies by Rekhi et al. and Høgdall et al., both with larger groups of clear-cell carcinoma patients (55 and 46, respectively), there were no cases of WT1-positive clear-cell ovarian cancer [17, 21]. There was also a divergence between our results and other authors' regarding the percentages of endometrioid ovarian tumors expressing WT1. We reported WT1 immunoreactivity in $45 \%$ of these carcinomas, and similar results were obtained by Cathro et al. (36\% of endometrioid EOCs). However, Hylander et al. [16] and Acs et al. [18] found no WT1-positive endometrioid tumors, while Høgdall et al. [21] reported WT1 immunoreactivity in only $5 \%$ of endometrioid EOCs. Reasons for these divergent results may lie in the sizes of study population in the reported studies, which had relatively small groups of patients with non-serous tumors; the use of differing immunohistochemical analysis protocols or antibodies, and the lack of a consistent definition of positive WT1 immunoreactivity. However, our findings concur with other authors when we postulate that serous EOCs are mainly WT1-positive tumors, while mucinous carcinomas present with either no or minor WT1 immunoreactivity.

Subsequently, we showed that WT1-positive patients were more likely to have lymph node metastases than WT1-negative patients $(24.2 \%$ vs. $5 \%$, respectively). Several earlier published studies concur with our finding that the presence of WT1 immunoreactivity is associated with metastatic lymph nodes, and that it correlates with an advanced stage of the disease [17, 22-25]. In a study by Liu et al., WT1 expression was associated with an aggressive phenotype of ovarian cancer [22]. In a pilot project directed by the US National Cancer Institute, WT1 was identified as one of the most important antigens for the production of cancer vaccines [26]. Clinical trials on vaccines against WT1 in ovarian cancer showed promising results and may lead to their use in future treatments [27, 28]. Furthermore, finding patients with more aggressive tumors (with positive WT1 immunoreactivity) may contribute to an improved selection of patients for immunotherapy.

Although the prognostic and immunotherapeutic roles of WT1 have been demonstrated in a variety of non-gynecological cancer types [8,9], the prognostic value of WT1 immunoreactivity in ovarian cancer remains unclear. We evaluated the prognostic value of WT1 in various histopathological types of EOC, and we found improved OS in patients with WT1-negative tumors; however, the finding was not statistically significant; and this finding is in line with those of several other studies [11, 16, 21]. However, in the subgroup of non-serous EOCs, we found that WT1 immunoreactivity was associated with significantly shortened OS. In a study by Hylander et al., patients with WT1-positive tumors had a shorter median OS than patients with WT1-negative tumors, but this difference was not statistically significant [16]. The authors suggested that the lack of a significant correlation between WT1 and survival may reflect the fact that most patients $(85 \%)$ were in an advanced stage of the disease [16]. This may also apply to our study, where over $80 \%$ of the WT1-positive patients were diagnosed at either FIGO stage III or IV of the disease. On the other hand, the large Danish 'MALOVA' ovarian cancer study of 560 ovarian cancer patients didn't find any association between WT1 immunoreactivity and disease specific survival when analyzing the whole study group as well considering a subgroup of 214 patients with serous EOC stage III [21]. Similarly, we did not find any association between WT1 immunoreactivity and OS in those patients with serous EOC. Yamamoto et al. examined serous EOC and classified the WT1-positive tumor as having both low and high levels of immunoreactivity. Interestingly in that study, in a group with high-levels of WT1 immunoreactivity, the patient outcomes were significantly worse than those in a group with low-level WT1 immunoreactivity (5-year survival rates of 36.5\% vs. $63.8 \%$, respectively). In a large meta-analysis by Lu et al., it was reported that WT1 overexpression did not have an unfavorable effect on overall survival in ovarian cancer, which is in line with our results [11].

We also found that in WT1-positive patients with non-serous EOC the OS was significantly shorter than in WT1-negative patients; however, the relatively small number of patients with these types of tumors in our study may be a limiting factor. Furthermore, the retrospective character of our study is another limitation. Of note, the treatment (surgery, chemotherapy) of our patients was not dependent on WT1 immunoreactivity, thus we conclude that the retrospective design did not influence the results. On the other hand, the experienced team of both pathologist and surgeons standardized and automated immunohistochemical staining and the evaluation of an established end-point (overall survival) are the main advantages of our study.

To the best of our knowledge, there is no study analyzing the association between WT1 immunoreactivity and patient survival in respect of various histopathological types of ovarian cancer; and this is important because these histopathological subtypes are molecularly and clinically different. The divergence of results regarding the prognostic role of WT1 across different studies may be partly explained by the fact that histological subtypes differ in their 
rates of WT1 expression. However, this issue requires further study.

In summary, our study indicates that immunohistochemical staining for WT1 helps in typing primary epithelial serous carcinomas; however, expression of the WT1 protein is not only limited to serous ovarian carcinoma. The results are suggestive of an association between the presence of WT1 and an aggressive clinical condition in ovarian cancer, yet it cannot be considered as a prognostic factor in EOC. Moreover, further studies on the association between WT1 expression and non-serous types of EOCs are required. In future, the targeting of WT1 could have had therapeutic implications in the treatment of this disease.

\section{Conflicts of interest}

The authors declare that they have no conflicts of interest.

\section{Financial disclosure}

The study was supported by the grant from the Centre of Postgraduate Medical Education, Warsaw, Poland (No. 98/2020).

\section{Acknowledgement}

We would like also to thank Robert Garrett for his assistance with the manuscript.

\section{References}

1. Bray F, Ferlay J, Soerjomataram I, et al. Global cancer statistics 2018: GLOBOCAN estimates of incidence and mortality worldwide for 36 cancers in 185 countries. CA Cancer J Clin. 2018; 68(6): 394-424, doi: 10.3322/caac.21492, indexed in Pubmed: 30207593.

2. Vecchia CLa. Ovarian cancer. Eur J Cancer Prev. 2017; 26(1): 55-62, doi: 10.1097/cej.0000000000000217, indexed in Pubmed: 26731563.

3. Rizzuto I, Behrens RF, Smith LA, et al. Risk of ovarian cancer in women treated with ovarian stimulating drugs for infertility. Cochrane Database Syst Rev. 2013; 6(8): CD008215, doi: 10.1002/14651858.CD008215.pub2, indexed in Pubmed: 23943232.

4. Torre LA, Trabert B, DeSantis CE, et al. Ovarian cancer statistics, 2018. CA Cancer J Clin. 2018; 68(4): 284-296, doi: 10.3322/caac.21456, indexed in Pubmed: 29809280.

5. Şakirahmet Şen D, Gökmen Karasu AF, Özgün Geçer M, et al. Utilization of Wilms' tumor 1 antigen in a panel for differential diagnosis of ovarian carcinomas. Turk J Obstet Gynecol. 2016; 13(1): 37-41, doi: 10.4274/tjod.22220, indexed in Pubmed: 28913087.

6. Sugiyama H. Wilms' tumor gene WT1: its oncogenic function and clinical application. Int J Hematol. 2001; 73(2): 177-187, doi: 10.1007/BF02981935, indexed in Pubmed: 11372729.

7. Pritchard-Jones K, Fleming S, Davidson D, et al. The candidate Wilms' tumour gene is involved in genitouri- nary development. Nature. 1990; 346(6280): 194-197, doi: 10.1038/346194a0, indexed in Pubmed: 2164159.

8. Charles AK, Mall S, Watson J, et al. Expression of the Wilms' tumour gene WT1 in the developing human and in paediatric renal tumours: an immunohistochemical study. Mol Pathol. 1997; 50(3): 138-144, doi: 10.1136/mp.50.3.138, indexed in Pubmed: 9292148.

9. Sagiyama H. WT1 (Wilms' Tumor Gene 1): Biology and Cancer Immunotherapy. Jpn J ClinOncol. 2010; 40(5): 377-87.

10. Carter JH, Deddens JA, Mueller G, et al. Transcription factors WT1 and p53 combined: a prognostic biomarker in ovarian cancer. Br J Cancer. 2018; 119(4): 462-470, doi: 10.1038/ s41416-018-0191-x, indexed in Pubmed: 30057405.

11. Lu J, Gu Y, Li Q, et al. Wilms' tumor 1 (WT1) as a prognosis factor in gynecological cancers: A meta-analysis. Medicine (Baltimore). 2018; 97(28): e11485, doi: 10.1097/ MD.000000000011485, indexed in Pubmed: 29995811.

12. Pecorrelli S, Creasman WT, Pettersson F, et al. FIGO annual report on the results of treatment in gynecologic cancer. J Epidemiol Biostat. 1998; 3: 75-102.

13. Seidman JD, Kurman RJ. Ovarian serous borderline tumors: a critical review of the literature with emphasis on prognostic indicators. Hum Pathol. 2000; 31(5): 539-557, doi: 10.1053/ hp.2000.8048, indexed in Pubmed: 10836293.

14. Thigpen T, Brady MF, Omura GA, et al. Age as a prognostic factor in ovarian carcinoma. The Gynecologic Oncology Group experience. Cancer. 1993; 71(2 Suppl): 606-614, doi: 10.1002/cncr.2820710218, indexed in Pubmed: 8420683.

15. Harmon RL, Sugarbaker PH. Prognostic indicators in peritoneal carcinomatosis from gastrointestinal cancer. Int Semin Surg Oncol. 2005; 2(1):3, doi: 10.1186/1477-7800-2-3, indexed in Pubmed: 15701175.

16. Hylander B, Repasky E, Shrikant P, et al. Expression of Wilms tumor gene (WT1) in epithelial ovarian cancer. Gynecol Oncol. 2006; 101(1): 12-17, doi: 10.1016/j.ygyno.2005.09.052, indexed in Pubmed: 16263157.

17. Rekhi B, Deodhar KK, Menon S, et al. Napsin A and WT 1 are useful immunohistochemical markers for differentiating clear cell carcinoma ovary from high-grade serous carcinoma. APMIS. 2018; 126(1): 45-55, doi: 10.1111/apm.12784, indexed in Pubmed: 29266433.

18. Acs G, Pasha T, Zhang PJ. WT1 is differentially expressed in serous, endometrioid, clear cell, and mucinous carcinomas of the peritoneum, fallopian tube, ovary, and endometrium. Int J Gynecol Pathol. 2004; 23(2): 110-118, doi: 10.1097/00004347200404000-00004, indexed in Pubmed: 15084838.

19. Cathro HP, Stoler MH. The utility of calretinin, inhibin, and WT1 immunohistochemical staining in the differential diagnosis of ovarian tumors. Hum Pathol. 2005; 36(2): 195-201, doi: 10.1016/j.humpath.2004.11.011, indexed in Pubmed: 15754297.

20. Goldstein NS, Uzieblo A. WT1 immunoreactivity in uterine papillary serous carcinomas is different from ovarian serous carcinomas. Am J Clin Pathol. 2002; 117(4): 541-545, doi: 10.1309/K84K-005F-TCB8-FV4B, indexed in Pubmed: 11939727.

21. Høgdall EVS, Christensen L, Kjaer SK, et al. Expression level of Wilms tumor 1 (WT1) protein has limited prognostic value in epithelial ovarian cancer: from the Danish „MALOVA" ovarian cancer study. Gynecol Oncol. 2007; 106(2): 318-324, doi: 10.1016/j.ygyno.2007.03.043, indexed in Pubmed: 17540436.

22. Liu Z, Yamanouchi K, Ohtao T, et al. High levels of Wilms' tumor 1 (WT1) expression were associated with aggressive 
clinical features in ovarian cancer. Anticancer Res. 2014; 34(5): 2331-2340, indexed in Pubmed: 24778040.

23. Rhodes A, Vallikkannu N, Jayalakshmi P. Expression of WT1 and PAX8 in the epithelial tumours of Malaysian women with ovarian cancer. Br J Biomed Sci. 2017; 74(2): 65-70, doi: 10.1080/09674845.2016.1220709, indexed in Pubmed: 28367736.

24. Yamamoto S, Tsuda H, Kita T, et al. Clinicopathological significance of WT1 expression in ovarian cancer: a possible accelerator of tumor progression in serous adenocarcinoma. Virchows Arch. 2007; 451(1): 27-35, doi: 10.1007/s00428-0070433-4, indexed in Pubmed: 17594113.

25. Barbolina MV, Adley BP, Shea LD, et al. Wilms tumor gene protein 1 is associated with ovarian cancer metastasis and modulates cell invasion. Cancer. 2008; 112(7): 1632-1641, doi: 10.1002/cncr.23341, indexed in Pubmed: 18260155.
26. Cheever MA, Allison JP, Ferris AS, et al. The prioritization of cancer antigens: a national cancer institute pilot project for the acceleration of translational research. Clin Cancer Res. 2009; 15(17): 5323-5337, doi: 10.1158/1078-0432.CCR09-0737, indexed in Pubmed: 19723653.

27. Vermeij R, Daemen T, de Bock GH, et al. Potential target antigens for a universal vaccine in epithelial ovarian cancer. Clin Dev Immunol. 2010; 2010, doi: 10.1155/2010/891505, indexed in Pubmed: 20885926.

28. Li G, Zeng Y, Chen X, et al. Human ovarian tumour-derived chaperone-rich cell lysate (CRCL) elicits $\mathrm{T}$ cell responses in vitro. Clin Exp Immunol. 2007; 148(1): 136-145, doi: 10.1111/j.1365-2249.2007.03323.x, indexed in Pubmed: 17349014 .

Submitted: 19 March, 2020

Accepted after reviews: 13 September, 2020 Available as AoP: 22 September, 2020 